\title{
Use of dental MRI for radiation-free guided dental implant planning: a prospective, in vivo study of accuracy and reliability
}

\author{
Tim Hilgenfeld ${ }^{1}$ (D) - Alexander Juerchott ${ }^{1} \cdot$ Johann M. E. Jende $^{1} \cdot$ Peter Rammelsberg $^{2} \cdot$ Sabine Heiland $^{1}$. \\ Martin Bendszus ${ }^{1}$. Franz S. Schwindling ${ }^{2}$
}

Received: 6 June 2020 / Revised: 20 July 2020 / Accepted: 4 September 2020 / Published online: 22 September 2020

(C) The Author(s) 2020

\begin{abstract}
Objectives To evaluate the accuracy and reliability of dental MRI for static guided implant surgery planning.

Materials and methods In this prospective study, a 0.4-mm isotropic, artifact-suppressed, 3T MRI protocol was used for implant planning and surgical guide production in participants in need of dental implants. Two dentists decided on treatment plan. Surgical guides were placed intraorally during a subsequent reference cone beam computed tomography (CBCT) scan. Interrater and inter-modality agreement were assessed by Cohen's kappa. For each participant, dental MRI and CBCT datasets were co-registered to determine three-dimensional and angular deviations between planned and surgically guided implant positions. Results Forty-five implants among 30 study participants were planned and evaluated (17 women, 13 men, mean age $56.9 \pm$ 13.1 years). Inter-rater agreement (mean $\kappa 0.814$; range $0.704-0.927$ ) and inter-modality agreement (mean $\kappa 0.879$; range $0.782-$ 0.901 ) were both excellent for the dental MRI-based treatment plans. Mean three-dimensional deviations were $1.1 \pm 0.7$ (entry point) and $1.3 \pm 0.7 \mathrm{~mm}$ (apex). Mean angular deviation was $2.4 \pm 1.5^{\circ}$. CBCT-based adjustments of MRI plans were necessary for implant position in $29.5 \%$ and for implant axis in $6.8 \%$ of all implant sites. Changes were larger in the group with shortened dental arches compared with those for tooth gaps. Except for one implant site, all guides were suitable for clinical use.

Conclusion This feasibility study indicates that dental MRI is reliable and sufficiently accurate for surgical guide production. Nevertheless, more studies are needed to increase its accuracy before it can be used for implant planning outside clinical trials. Key Points

- An excellent reliability for the dental MRI-based treatment plans as well as agreement between dental MRI-based and CBCTbased (reference standard) decisions were noted.

- Ideal implant position was not reached in all cases by dental MRI plans.

- For all but one implant site surgical guides derived from dental MRI were sufficiently accurate to perform implant placement (mean three-dimensional deviations were $1.1 \pm 0.7$ (entry point) and $1.3 \pm 0.7 \mathrm{~mm}$ (apex); mean angular deviation was $2.4 \pm$ $\left.1.5^{\circ}\right)$.
\end{abstract}

Keywords Magnetic resonance imaging $\cdot$ Cone beam computed tomography $\cdot$ Dental implants $\cdot$ Humans $\cdot$ Tooth

Abbreviations

CBCT Cone beam computed tomography

CI Confidence interval

К Cohen's kappa

Tim Hilgenfeld

tim.hilgenfeld@med.uni-heidelberg.de

1 Department of Neuroradiology, Heidelberg University Hospital, Im Neuenheimer Feld 400, 69120 Heidelberg, Germany

2 Department of Prosthodontics, Heidelberg University Hospital, Heidelberg, Germany

\section{Introduction}

Dental implants were introduced 50 years ago [1] and are now an established treatment option for the replacement of missing teeth [2]. In the USA, an adjusted increase in the prevalence of dental implants of $14 \%$ per year has been recorded, rising from $0.7 \%$ in $1999 / 2000$ to $5.7 \%$ in $2015 / 2016$ [3]. Dental implants can be planned by using either plain radiographs or cone beam computed tomography (CBCT) or computed tomography (CT). Prosthetically driven backward planning, also known as guided implant surgery, is currently of fundamental importance in implant surgery [4]. In this method, 
the three-dimensional (3D) position of the implant is determined by the optimum design of the future prosthetic restoration (e.g., the implant-supported single crown) and transferred into the patient by use of a surgical guide. To define the optimum prosthetically related implant position within the limits of available alveolar bone, $3 \mathrm{D}$ imaging is required $[5,6]$. As the use of dental implants grows, the number of CBCT and CT examinations will also increase [7]. Compared with twodimensional radiography like panoramic radiographs, however, the radiation dose of CBCT is nonetheless 2-200 times higher (range 10-1000 $\mu \mathrm{Sv}$; effective CBCT dose in this study, $211 \mu \mathrm{Sv}$ ) $[8,9]$. A meta-analysis identified the potential lifetime risks for thyroid cancer and meningioma posed by repeated X-ray-based imaging (two- and three-dimensional images) in dentistry [10]. In this context, dental MRI as a non-ionizing, cross-sectional imaging modality proofed to be a promising alternative for the two-dimensional evaluation/ planning of implant sites as performed with panoramic radiographs, with a predefined implant position [11-13]. Previous studies concluded that measurement errors of dental MRI and $\mathrm{CT}$ are comparable for height and width measurements of jaw bones [13-16]. In prosthetically driven backward planning, however, the implant position is defined within the image dataset and isotropic imaging, as made available by CBCT, is necessary. The feasibility of dental MRI for backward planning has been show very recently in a case series [17]. The reliability and accuracy of dental MRI, however, has not yet been evaluated in a clinical setting. We wanted to test, therefore, whether implant planning decisions based on dental MRI would differ from those based on the reference imaging technique of CBCT and whether surgical guides derived from dental MRI would be sufficiently accurate to perform implant placement. The objectives of this study were therefore (I) to qualitatively evaluate the reliability and accuracy of dental MRI-based decisions regarding implant planning and (II) to quantitatively evaluate the accuracy of dental MRI-based surgical guides.

\section{Materials and methods}

\section{Study sample}

This prospective study was approved by the institutional ethics committee of Heidelberg University Hospital (approval number S-404/2014). Written informed consent was obtained from all participants. Potentially eligible participants were identified by means of a clinical examination, and 34 participants were consecutively enrolled in total (Fig. 1). The inclusion criteria were as follows: in need of a dental implant (including implant placement with simultaneous bone augmentation), teeth in both quadrants of the jaw (minimal number of three) to allow for reliable guide positioning, delayed implantation (at least 3 months after tooth extraction), and a stable medical condition to undergo implant surgery. The following criteria were grounds for exclusion: a two-stage surgical procedure with separate bone augmentation and implant insertion, contraindications to 3T MRI, age below 18 years, pregnancy, and claustrophobia. The study was planned and performed according to the STARD guidelines [18].

\section{Planning procedure for implant placement}

Full-arch impressions (Impregum, 3M GmbH) to obtain a stone cast and a $0.4-\mathrm{mm}$ isotropic, artifact-suppressed, proton-weighted dental MRI scan of the relevant jaw were taken for each participant (Fig. 2). To enable accurate segmentation of tooth surfaces in MRI data, a splint technique was used during the dental MRI examination, as described previously [19]. DICOM data from the dental MRI and STL data from the digitalized stone cast (D2000, 3shape) were subsequently imported and co-registered in an established software program for guided implant surgery (coDiagnostiX 9.12, Dental Wings Inc.). To assess the intra-modality reliability, backward planning was performed independently by two experienced dentists with more than 7 and 20 years' experience of implant planning, respectively. Differences between decisions were clarified in a consensus reading. The dentists were asked to determine a treatment plan (implant type and dimensions, need for and type of bone augmentation, and implant position and axis) in accordance with manufacturer specification of the implants and established clinical criteria [20,21]. If bone augmentation was required, the type of augmentation had to be specified (spread, split, bone chips, block, sinus lift, condensing, or any combination of the aforementioned procedures). After selecting the optimum implant position and axis, a tooth-supported guide with a thickness of $3 \mathrm{~mm}$ was designed, exported as STL, and 3D printed for each participant (Pro2, Asiga). Finally, each participant underwent a CBCT scan for the purposes of guided implant planning.

\section{Image acquisition parameters}

Dental MRI examinations were performed with a 3T MRI system (Magnetom Tim-Trio, Siemens Healthcare) using a dedicated 15-channel dental coil (Mandibula, Noras MRI products $\mathrm{GmbH}$ ). A multi-slab acquisition with view-angle tilting gradient was used, based on a sampling perfection with application-optimized contrasts using different flip-angle evolution (MSVAT-SPACE) prototype sequence (repetition time $1170 \mathrm{~ms}$, echo time $6.4 \mathrm{~ms}$; field of view $168 \times 131 \mathrm{~mm}^{2}$; voxel size $0.4 \times 0.4 \times 0.4 \mathrm{~mm}^{3}$; matrix $384 \times 300$; slice oversampling $220 \%$; slices 80 ; acquisition time $7.45 \mathrm{~min}$ ). This MRI technique uses slab-selective excitation and refocusing radiofrequency pulses that enable interleaved multi-slab acquisition [22]. The technique was specifically 


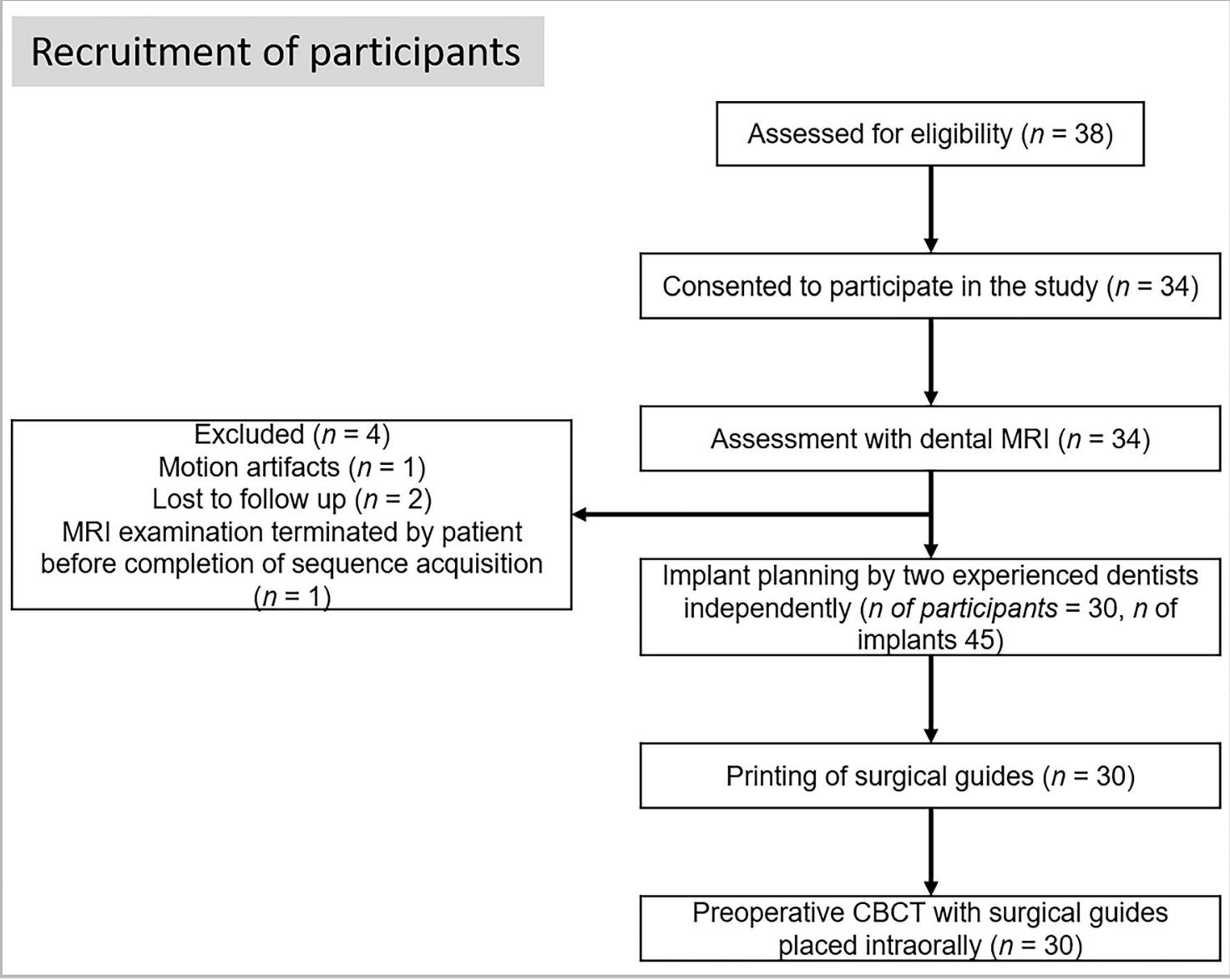

Fig. 1 Flow chart illustrating the recruitment of participants

optimized and evaluated for high spatial resolution, artifactsuppressed dental MRI, as described previously [23].

CBCT imaging (3D Accuitomo 170, J Morita) was performed as follows: field of view $8 \times 8 \mathrm{~cm}^{2}$; tube voltage $90 \mathrm{kV}$; tube current $7 \mathrm{~mA} ; 14$ bit; $360^{\circ}$ rotation in $17 \mathrm{~s} ; 560$ frames; and an isotropic voxel size of $160 \mu \mathrm{m}$.

\section{Accuracy evaluation}

To transfer the MRI-based implant positions and axes into the CBCT datasets, surgical guides with a metal marker were placed intraorally during the CBCT scans (Fig. 2). Wooden spatulas were additionally placed intraorally to ensure a stable support of all opposite teeth on the surgical guide. The qualitative accuracy was assessed by re-evaluation of MRIderived implant position and the MRI-derived treatment plan in CBCT by both dentists together, according to the same criteria they were using for the MRI-based planning. For the quantitative accuracy analysis, the CBCT data were imported into the same implant planning software and co-registered with the dental MRI using the tooth surfaces as references.
The marker within the surgical guide on the CBCT examinations was used to identify the surgically guided implant position. Afterwards, the planned implant position in the dental MRI datasets was compared with the surgically guided implant position in the CBCT images (Fig. 2). Finally, the 3D deviation of the entry point and implant apex were calculated, as was the deviation of the implant axis.

\section{Statistical analysis}

Because this is a prospective feasibility study, no sample size calculation was possible, and $p$ values are descriptive in nature. To determine inter-rater and inter-modality reliability, Cohen's kappa ( $\mathrm{K}$ ) and the $95 \%$ confidence interval (CI) were used and interpreted as described previously [24]. Data were analyzed using SPSS (version 25, 2017, SPSS Inc.). Differences in accuracy between implant sites used to restore shortened dental arches and those in tooth gaps were analyzed using Mann-Whitney $U$ or two-tailed Student $t$ test, depending on data distribution in Shapiro-Wilk test. A level of significance was defined at $p \leq 0.05$. 


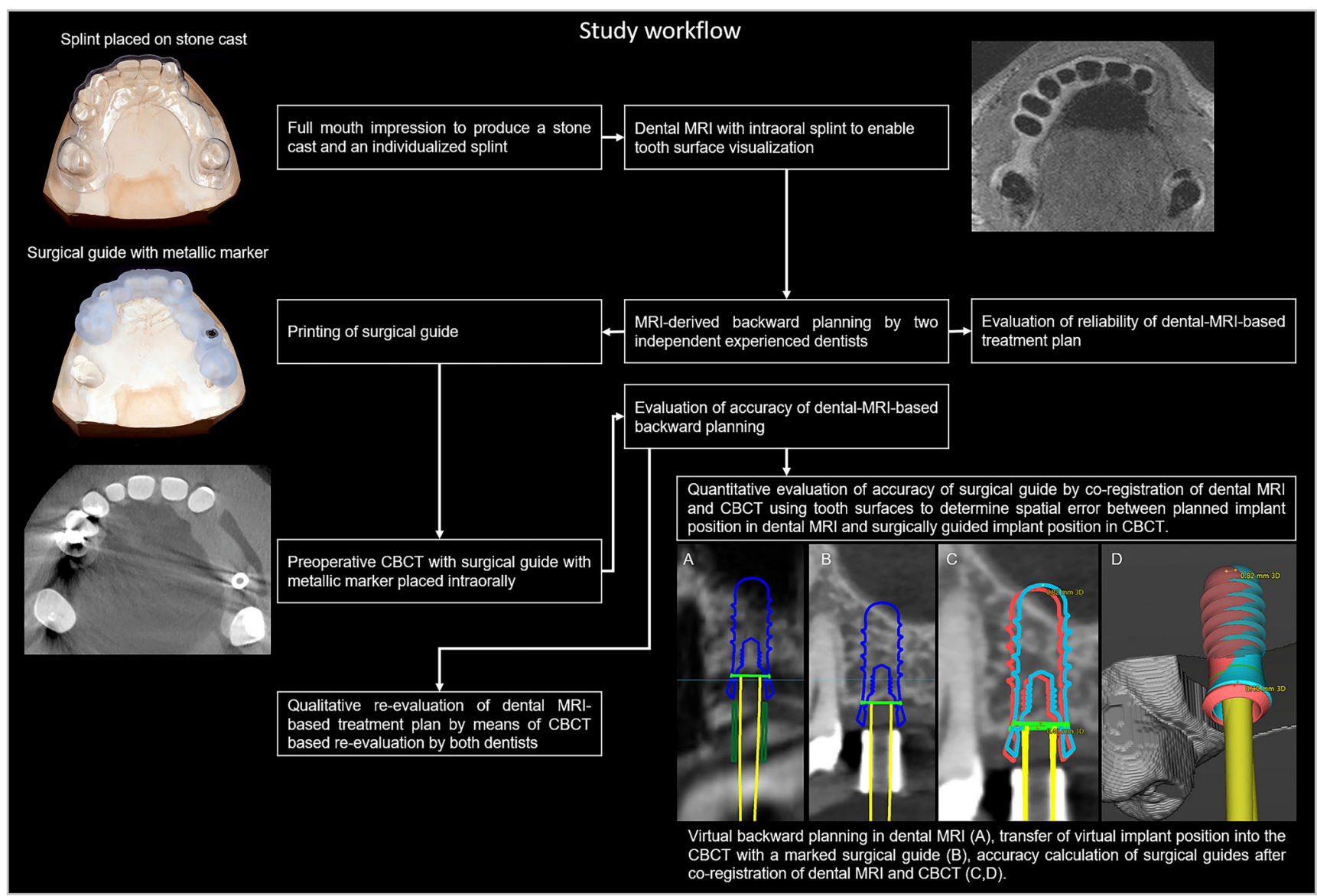

Fig. 2 Flow chart illustrating the workflow of the study

\section{Results}

\section{Participant characteristics}

In total, 34 participants were consecutively enrolled in this study. Four participants could not be included in the final analysis (Fig. 1). Thus, 45 implant sites among 30 participants were planned and evaluated in total ( 17 women, 13 men, mean age $56.9 \pm 13.1$ years). Implants were placed between neighboring teeth in 26 sites and used to restore shortened dental arches in 19 sites (i.e., implant splints were supported distally by soft tissue only).

\section{Planning accuracy of dental MRI}

The high spatial resolution protocol produced detailed images of the jaws that could be assessed in all three dimensions without a reduction in image quality (Fig. 3). Consequently, mean inter-rater agreement (mean $\mathrm{k} 0.814$; $95 \%$ CI $0.741-$ 0.887 ; Fig. $4 \mathrm{a}$ ) and mean inter-modality agreement between dental MRI-based and CBCT-based decisions (mean к 0.879; 95\% CI 0.811-0.948) were both excellent. After re-evaluation of the dental MRI-based plans by means of comparison with CBCT, no changes were made with regard to implant type, neck type, implant length, or implant diameter for 43 out of 45 planned implant sites (inter-modality $\mathrm{K}$ range $0.788-1$; Fig. $4 a)$. For one site, the implant diameter and neck design were changed (from 4.1 to $4.8 \mathrm{~mm}$ and from a regular neck to a wide neck, respectively). With regard to another site, an initial implant length of $8 \mathrm{~mm}$ was changed to $10 \mathrm{~mm}$. Bone augmentation procedures were performed for more than $50 \%$ of implant sites ( 23 out of 45 ; Table 1). For 42 of the 45 implant sites, the decision whether to perform bone augmentation or not was made correctly on the basis of dental MRI planning ( $\mathrm{k} 0.867 ; 95 \%$ CI $0.722-1$ ). For three sites, the need for bone augmentation was not predicted by dental MRI, but was subsequently identified based on CBCT images (negative predictive value (NPV) 0.88; see example in Fig. 5a). For one implant site, extensive bone loss was predicted by dental MRI correctly. Consequently, the implant site was classified as unsuitable for simultaneous bone augmentation and implant placement (Fig. 5b). Dental MRI-based planning did not incorrectly predict the need for bone augmentation for any site (based on re-evaluation after CBCT; positive predictive value (PPV) 1). For all implant sites where the need for bone augmentation was identified on the basis of dental MRI $(n=20)$, the decision was confirmed by CBCT ( 20 out of 20 sites; $\kappa$ for all 23 augmented sites $=0.782 ; 95 \%$ CI $0.588-0.976$ ). 
Table 1 Overview of all planned implant sites and their respective accuracy of surgically guided implant position

\begin{tabular}{|c|c|c|c|c|c|c|c|c|c|c|}
\hline \multirow{2}{*}{$\begin{array}{l}\text { Patient } \\
\text { Number }\end{array}$} & \multicolumn{2}{|l|}{ Implant site } & \multirow[t]{2}{*}{$\begin{array}{l}\text { Kennedy } \\
\text { class }\end{array}$} & \multicolumn{2}{|c|}{ Bone augmentation } & \multicolumn{2}{|c|}{$\begin{array}{l}\text { Corrections after } \\
\text { CBCT }\end{array}$} & \multicolumn{3}{|c|}{ Accuracy of MRI-derived surgical guide } \\
\hline & $\begin{array}{l}\text { Neighboring } \\
\text { teeth }\end{array}$ & Position & & $\begin{array}{l}\text { Based on } \\
\text { MRI }\end{array}$ & $\begin{array}{l}\text { Based on } \\
\text { CBCT }\end{array}$ & $\begin{array}{l}\text { Position } \\
\text { of } \\
\text { entry } \\
\text { point } \\
{[\mathrm{mm}]}\end{array}$ & $\begin{array}{l}\text { Angulation } \\
{\left[{ }^{\circ}\right]}\end{array}$ & $\begin{array}{l}\text { 3D deviation at entry } \\
\text { point }[\mathrm{mm}]\end{array}$ & $\begin{array}{l}\text { 3D deviation at } \\
\text { apex }[\mathrm{mm}]\end{array}$ & $\begin{array}{l}\text { Angular } \\
\text { deviation }\left[^{\circ}\right]\end{array}$ \\
\hline \multirow[t]{2}{*}{1} & Free-ending & 15 & 1 & Sinus lift & Sinus lift & 2.0 & 10 & 1.3 & 1.3 & 1.6 \\
\hline & Free-ending & 26 & 1 & Sinus lift & Sinus lift & 1.0 & - & 1.2 & 1.4 & 2.2 \\
\hline \multirow[t]{2}{*}{2} & Free-ending & 36 & 1 & No & No & - & - & 1.8 & 2.1 & 3.8 \\
\hline & Free-ending & 46 & 1 & No & No & - & - & 0.4 & 1.2 & 4.7 \\
\hline \multirow[t]{2}{*}{3} & Free-ending & 45 & 2 & No & No & 1.0 & - & 1.3 & 1.9 & 3.7 \\
\hline & Free-ending & 47 & 2 & No & No & - & - & 2.1 & 3.1 & 6.6 \\
\hline \multirow[t]{2}{*}{4} & Free-ending & 14 & 2 & No & No & 8.0 & - & 0.9 & 1.0 & 1.3 \\
\hline & Free-ending & 16 & 2 & Sinus lift & Sinus lift & - & - & 0.3 & 0.5 & 1.1 \\
\hline 5 & Free-ending & 26 & 2 & Sinus lift & Sinus lift & 1.0 & - & 1.8 & 2.0 & 1.3 \\
\hline \multirow[t]{2}{*}{6} & Free-ending & 35 & 2 & No & No & - & - & 0.2 & 0.2 & 0.0 \\
\hline & Free-ending & 37 & 2 & $\begin{array}{l}\text { Bone } \\
\text { chips }\end{array}$ & Bone chips & - & - & 0.5 & 0.7 & 1.4 \\
\hline \multirow[t]{2}{*}{7} & Free-ending & 25 & 2 & Sinus lift & Sinus lift & - & - & 0.4 & 0.8 & 3.2 \\
\hline & Free-ending & 27 & 2 & Sinus lift & Sinus lift & - & - & 0.3 & 0.5 & 1.7 \\
\hline 8 & Free-ending & 37 & 2 & No & $\begin{array}{l}\text { Bone } \\
\text { condens- } \\
\text { ing }\end{array}$ & 1.0 & 10 & 0.8 & 1.1 & 2.0 \\
\hline \multirow[t]{2}{*}{9} & Free-ending & 44 & 2 & No & No & 2.0 & & 0.7 & 0.9 & 2.5 \\
\hline & Free-ending & 47 & 2 & No & No & 1.0 & 10 & 1.3 & 1.6 & 2.5 \\
\hline 10 & Free-ending & 37 & 2 & No & No & - & - & 2.9 & 3.1 & 6.3 \\
\hline 11 & Free-ending & 46 & 2 & Bone split & Bone split & 1.5 & - & 1.2 & 1.6 & 2.8 \\
\hline \multicolumn{6}{|c|}{ Mean \pm standard deviation $[\mathrm{mm}]$} & $1 \pm 1.9$ & $1.7 \pm 3.8$ & $1.1 \pm 0.7$ & $1.4 \pm 0.8$ & $2.7 \pm 1.8$ \\
\hline 3 & Tooth gap & 36 & 2 & No & No & - & - & 1.1 & 1.3 & 2.9 \\
\hline \multirow[t]{2}{*}{11} & Tooth gap & 35 & 2 & No & No & - & - & 1.2 & 1.6 & 3.1 \\
\hline & Tooth gap & 37 & 2 & No & No & - & - & 1.7 & 2.1 & 3.7 \\
\hline \multirow[t]{2}{*}{12} & Tooth gap & 17 & 3 & No & No & - & - & 0.6 & 0.8 & 1.5 \\
\hline & Tooth gap & 25 & 3 & No & No & - & - & 0.9 & 1.1 & 1.2 \\
\hline \multirow[t]{2}{*}{13} & Tooth gap & 45 & 3 & Bone split & Bone split & - & - & 2.6 & 2.5 & 1.8 \\
\hline & Tooth gap & 47 & 3 & Bone split & Bone split & 2.0 & - & 3.0 & 2.5 & 4.3 \\
\hline 14 & Tooth gap & 26 & 3 & Sinus lift & Sinus lift & - & - & 0.6 & 1.0 & 3.0 \\
\hline 15 & Tooth gap & 36 & 3 & No & No & - & - & 1.0 & 1.1 & 0.8 \\
\hline \multirow[t]{2}{*}{16} & Tooth gap & 46 & 3 & Bone split & Bone split & - & - & 1.0 & 1.1 & 0.9 \\
\hline & Tooth gap & 47 & 3 & Bone split & Bone split & - & - & 1.1 & 1.1 & 0.0 \\
\hline 17 & Tooth gap & 15 & 3 & No & No & - & - & 2.0 & 1.6 & 3.8 \\
\hline 18 & Tooth gap & 21 & 3 & No & No & - & - & 0.4 & 0.4 & 0.0 \\
\hline 19 & Tooth gap & 45 & 3 & No & No & - & - & 0.7 & 0.8 & 1.1 \\
\hline 20 & Tooth gap & 36 & 3 & No & $\begin{array}{c}\text { Split and } \\
\text { chips }\end{array}$ & 1.0 & - & 0.6 & 0.7 & 1.4 \\
\hline \multirow[t]{2}{*}{21} & Tooth gap & 25 & 3 & Sinus lift & Sinus lift & - & - & 1.6 & 1.6 & 1.0 \\
\hline & Tooth gap & 26 & 3 & Sinus lift & Sinus lift & - & - & 1.7 & 1.6 & 1.6 \\
\hline 22 & Tooth gap & 47 & 3 & Bone split & Bone split & 1.0 & - & 0.5 & 0.5 & 2.5 \\
\hline 23 & Tooth gap & 14 & 3 & Sinus lift & Sinus lift & - & - & 0.5 & 1.3 & 4.4 \\
\hline 24 & Tooth gap & 47 & 3 & No & No & - & - & 0.9 & 1.1 & 1.9 \\
\hline 25 & Tooth gap & 26 & 3 & Sinus lift & Sinus lift & - & - & 0.9 & 1.0 & 1.4 \\
\hline 26 & Tooth gap & 36 & 3 & No & No & 1.0 & - & 2.5 & 2.7 & 3.1 \\
\hline 27 & Tooth gap & 26 & 3 & Sinus lift & Sinus lift & - & - & 0.6 & 0.9 & 2.7 \\
\hline 28 & Tooth gap & 36 & 3 & No & No & - & - & 0.6 & 1.0 & 2.4 \\
\hline 29 & Tooth gap & 46 & 3 & No & Bone split & - & - & 0.8 & 1.1 & 3.5 \\
\hline 30 & Tooth gap & 26 & 3 & Sinus lift & Sinus lift & - & - & 0.8 & 0.8 & 1.2 \\
\hline Mean $\pm \mathrm{st}$ & tandard deviation & {$[\mathrm{mm}]$} & & & & $0.2 \pm 0.5$ & $0 \pm 0$ & $1.1 \pm 0.7$ & $1.3 \pm 0.6$ & $2.1 \pm 1.3$ \\
\hline$p$ value (fi & ree-ending vs. to & th gap) & & & & $0.012^{\mathrm{a}}$ & $0.035^{\mathrm{a}}$ & $0.659^{\mathrm{a}}$ & $0.821^{\mathrm{a}}$ & $0.207^{\mathrm{b}}$ \\
\hline
\end{tabular}

${ }^{\text {a }}$ Mann-Whitney $U$ test

${ }^{\mathrm{b}}$ Two tailed Student $t$ test 
Fig. 3 Direct comparison of two examples of implant planning in dental MRI and CBCT. In example a, implant insertion was planned in region 36. Based on dental MRI and CBCT images, no bone augmentation was necessary. In addition, a large combined periodontal-endodontic lesion can be seen in region 37 in both imaging modalities. Note the good delineation of the tooth surfaces against the bright toothpaste (\#) in the splint (arrow). In example b, implant insertion was planned in regions 25 and 27 (coronal images from region 27). For both imaging modalities, it was decided that sinus lift augmentation was necessary in both regions
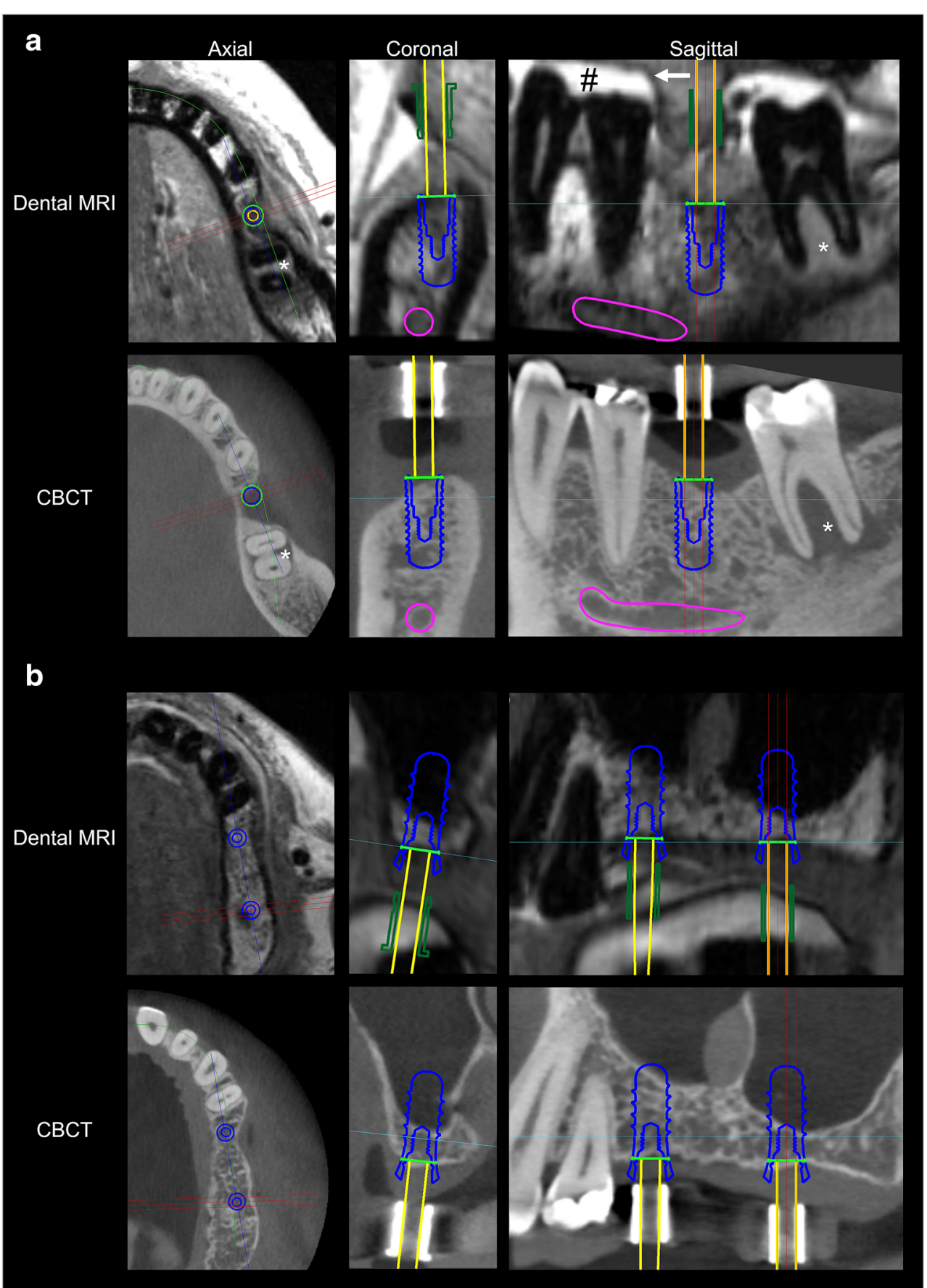

For 31 out of 44 sites $(70.5 \%)$, the planned implant position was not altered after the CBCT-based re-evaluation. Small changes were made to the implant position of 13 sites (29.5\%; mean change of $1.8 \mathrm{~mm}$ ) and to the implant axis of three sites $\left(6.8 \%\right.$; mean change of $\left.10^{\circ}\right)$. The subgroup analysis of implants placed in tooth gaps versus implants placed to restore shortened dental arches revealed significant differences (Table 1). Significantly less corrections were performed by both dentists for the implants placed in tooth gaps compared with implants placed in free-ending positions for both, the CBCT-based corrections of the entry point $(0.2 \pm$ 0.5 vs. $1 \pm 1.9 \mathrm{~mm} ; p=0.01)$ and angulation $\left(0 \pm 0^{\circ}\right.$ vs. $1.7 \pm$ $3.8 ; p=0.04)$. If the single $8 \mathrm{~mm}$ outlier in the free-end subgroup (in dental MRI-missed potential remainder of a tooth root measuring $1 \mathrm{~mm}$ in CBCT), however, is excluded, the accuracy of that subgroup $(0.6 \pm 1.9 \mathrm{~mm})$ is close the tooth gap subgroup and the mean change of all implants is reduced from 1.8 to $1.3 \mathrm{~mm}$. As all changes, except one, affected the 


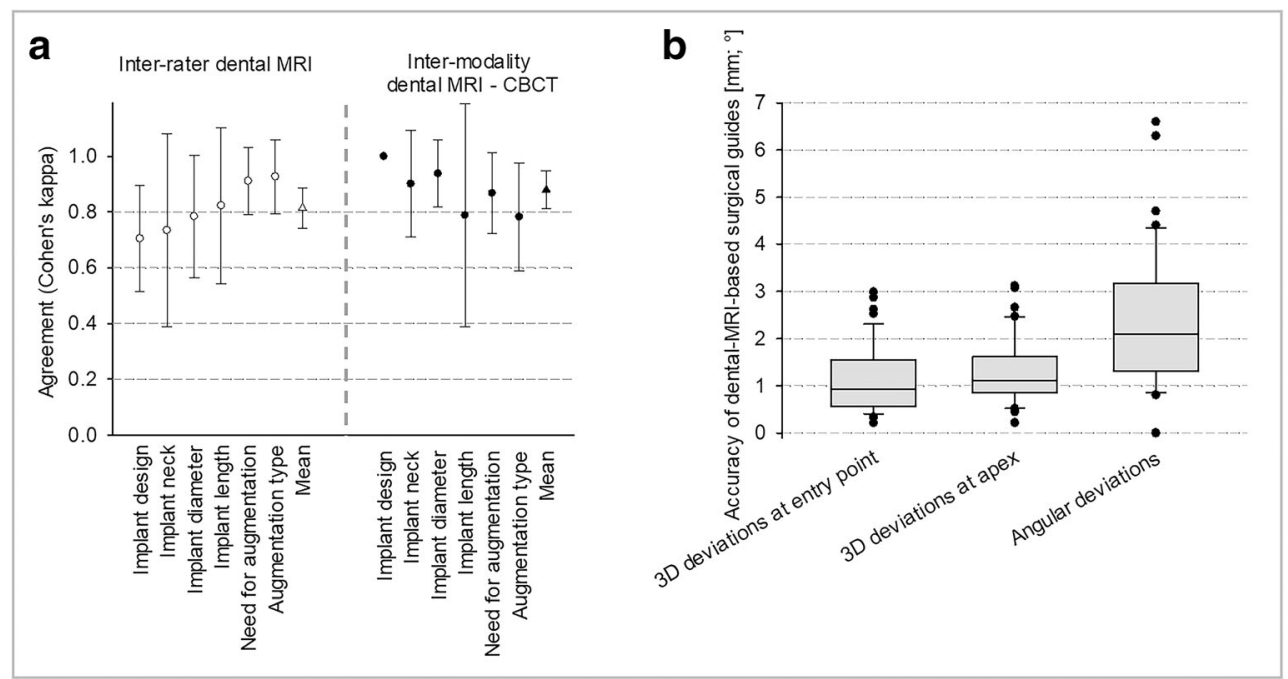

Fig. 4 Inter-rater reliability of dental MRI-based treatment plan and inter-modality accuracy of dental MRI-based decisions compared with CBCT (a). Accuracy of dental MRI-based surgical guides (b)

second (unguided) drill, and not the first (guided) drill, $97.8 \%$ of guides (43 out of 44 ) were classified as suitable for implant surgery after qualitative evaluation of dental MRI.

Evaluation of the quantitative accuracy of the dental MRIbased surgical guides for the 44 planned implants revealed mean 3D deviations of $1.1 \pm 0.7 \mathrm{~mm}$ at the entry point (minimum $0.2 \mathrm{~mm}$; maximum $3 \mathrm{~mm}$ ) and $1.3 \pm 0.7 \mathrm{~mm}$ at the implant apex (minimum $0.2 \mathrm{~mm}$; maximum $3.1 \mathrm{~mm}$; Fig. $4 \mathrm{~b}$ ). In addition, a mean angular deviation of $2.4 \pm 1.5^{\circ}$ (minimum $0^{\circ}$; maximum $6.6^{\circ}$ ) was observed. Slightly larger deviations were found for implant sites with shortened dental arches vs. implant sites in tooth gaps for the 3D deviation of entry point/implant apex, as well as the angulation, without reaching statistical significance (Table 1).

\section{Discussion}

This study shows that dental MRI-based backward planning is highly reliable and results in sufficiently accurate surgical guides. It also showed, however, that the method is not yet capable to achieve the highest prosthetic and surgical demands on treatment planning in all cases.

This study has several methodological strengths. First, it made use of a previously established technique to visualize tooth surfaces within dental MRI [19]. As a result, it was possible to integrate dental MRI into an existing digital workflow without the need for additional software or timeconsuming postprocessing steps. Second, it used a singlescan protocol with an examination time of less than $10 \mathrm{~min}$. This has the potential to reduce examination costs. Moreover, in contrast to the earlier studies from Gray et al and Pompa et al studies $[11,13]$, we voted for a dedicated dental coil instead of a standard head and neck coil. The higher signal- to-noise ratio $[25,26]$ allowed for smaller and isotropic voxel size (440 $\mu \mathrm{m}$ isotropic). The latter is a prerequisite for backward planning, as multiplanar reconstructions are essential for finding the correct implant axis in relation to available bone and prosthodontics demands at the same time. The chosen sequence differed slightly from Flügge et al who used a SPACE and not a MSVAT-SPACE sequence. The MSVATSPACE sequence, however, offered the advantage of $56 \%$ less susceptibility artifacts compared with the SPACE in a previous study [23]. This likely results in improved tooth surface reconstructions directly adjacent to metallic crowns, pontics, or implants which are frequently observed in patients in need of dental implants. Thereby, it contributes to increase the accuracy and applicability of dental MRI-based backward planning. Finally, the accuracy of the dental MRI-based treatment plans was directly compared with the clinical reference imaging modality of CBCT.

Although dental MRI-derived implant planning resulted in accurate decisions regarding implant type, dimensions, and type of bone augmentation for most implant sites, it must be noted that the planned implant position and angle were changed slightly (for $29.5 \%$ and $6.8 \%$ of implant sites, respectively) at the stage of unguiding drilling after CBCT re-evaluation. In addition, three participants in need of bone augmentation were not identified as such from dental MRI images (NPV 0.88; PPV 1). Without the CBCT for re-evaluation, this would have resulted in a moderate extension of operation time in two cases and in a failed implantation in one case. This might be because partially calcified tissues appear different in CBCT images than they do in MRI images, especially if cortical bone borders are still intact as in our case. Consequently, the volume of bone available can be overestimated from dental MRI images. Implant planning based on dental MRI might therefore require the involvement 
Fig. 5 Two cases of extensive, bone loss in dental MRI and CBCT. Based on dental MRI, case a was misclassified as sufficient bone without the need for bone augmentation. At implant site 36 , the preserved outer shape of the alveolar bone was misleading. In the second case (b), dentists correctly identified extensive bone loss in implant site 17 from the dental MRI examination. It was confirmed by CBCT that simultaneous implant insertion and bone augmentation were therefore not possible

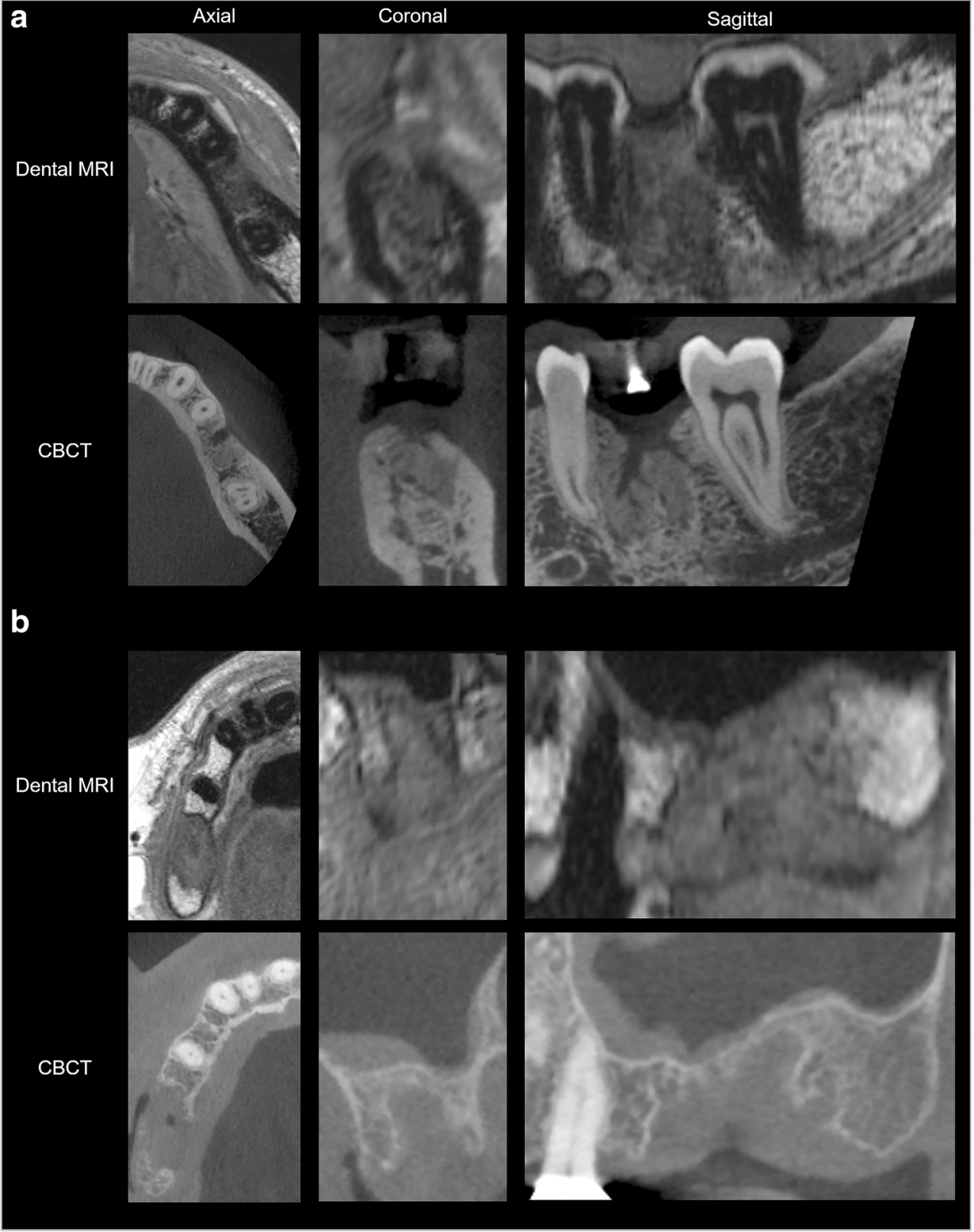

of dentists and oral surgeons with sufficient experience of interpreting dental MRI images or a learning curve has to be acknowledged, respectively. Moreover, other sequence techniques may offer improved evaluation of bone, like ultrashort or zero time of echo sequences [27]. However, other disadvantages may come along with such sequences like lower resolution, lower image quality, and more susceptibility artifacts compared with MSVAT-SPACE [23].

The subgroup analysis revealed more and significantly larger corrections of MRI-derived implant position/ angulation in free-ending positions compared with implant sites between neighboring teeth and slightly less accurate MRI-derived surgical guides (not statistically significant). As the spatial distribution of available teeth surfaces for co- registration is limited in patients with shortened arches, the coregistration of MRI and digitalized impression data might be less accurate in these patients, leading to a less precise transfer of the virtual implant position into the surgical guide. That result is in accordance with previous studies which reported a similar dependency of the accuracy and the number of residual teeth $[28,29]$.

The authors are not aware of any similar studies that have evaluated the accuracy of CBCT-based templates in vivo, most likely because of ethical concerns associated with a second preoperative CBCT scan. However, one ex vivo study by Kühl et al on CBCT-derived template accuracy is available for comparison [30]. Their study investigated the accuracy of surgical guides printed from cast models using the same planning 
and evaluation software ex vivo. For a 10-mm implant (the most frequently used implant length in our study), Kühl et al reported an apical deviation of $0.49 \mathrm{~mm}$ (minimum 0.13 , maximum $1.09 \mathrm{~mm}$ ). By comparison, apical deviation in our study was larger (mean $1.3 \pm 0.7$; minimum 0.2; maximum $3.1 \mathrm{~mm}$ ). This may be due to our in vivo setting (i.e., incorporating motion artifacts) and a lower scanning resolution $(0.4 \mathrm{~mm}$ isotropic; accuracy of optical scanner used by Kühl et al: approximately $15 \mu \mathrm{m})$. Another source of errors in our study was registration accuracy, which had an effect twice: once in the implant planning procedure (registration of dental MRI data with digitalized tooth models) and once in the quantitative assessment of surgical guide accuracy (registration of dental MRI with CBCT). For registration in implant planning, tooth surfaces are commonly used. The errors for in vivo tooth surface reconstructions derived from dental MRI and CBCT (mean error \pm root mean square of dental MRI and CBCT $0.26 \pm 0.1$ and $0.1 \pm 0.04 \mathrm{~mm}$, respectively) were reported in a recent study [19]. This explains, at least in part, why accuracy was lower in our study than in Kühl et al.

Several limitations of this particular application of dental MRI must be addressed. The value of our reliability assessment is limited to some extent, as both surgeons were working in the same department. Moreover, the costs of dental MRI currently restrict its clinical use.

In conclusion, our feasibility study contributes to the current literature by providing evidence that dental MRI-based backward planning is reliable and results in surgical guides sufficiently accurate for implant placement. More research, however, is necessary to increase the accuracy of dental MRI, for example, by increasing spatial resolution or decreasing acquisition time to reduce motion artifacts. These findings may help to facilitate prosthetically driven backward implant planning without radiation exposure. This is particularly important in relation to younger individuals, who are more sensitive to radiation. However, more studies regarding dental MRI and implant placement are required before this imaging modality can be used outside clinical studies.

Acknowledgments This project was supported by a grant from the ITI Foundation, Switzerland (grant number 1346 2018). S.H. and A.J. were supported in part by the Dietmar Hopp Stiftung (project number 23011228). We are thankful for the support of U.K. Deisenhofer during participant recruitment. The authors would like to thank Siemens Healthcare $\mathrm{GmbH}$, especially Mathias Nittka, $\mathrm{PhD}$, for their kind cooperation and assistance in setting up the MSVAT-SPACE sequence. Furthermore, we are grateful to Dental Wings Inc. for providing the treatment evaluation tool and waiving the guide fee. Finally, we would like to thank NORAS MRI products GmbH, especially Turgay Celik, for their research cooperation in developing dedicated dental coils. English language correction was performed by Hazel Davies, copy editor.

Funding Open Access funding provided by Projekt DEAL. This study has received funding by a grant from the ITI Foundation, Switzerland (grant number 1346_2018). S.H. and A.J. were supported in part by the Dietmar Hopp Stiftung (project number 23011228). Furthermore, we are grateful to Dental Wings Inc. for providing the treatment evaluation tool and waiving the guide fee.

\section{Compliance with ethical standards}

Guarantor The scientific guarantor of this publication is Tim Hilgenfeld.

Conflict of interest The authors of this manuscript declare no relationships with any companies, whose products or services may be related to the subject matter of the article.

Statistics and biometry No complex statistical methods were necessary for this paper.

Informed consent Written informed consent was obtained from all subjects (patients) in this study.

Ethical approval Institutional Review Board approval was obtained.

Study subjects or cohorts overlap Some study subjects or cohorts have been previously reported in:

Hilgenfeld T, Juerchott A, Deisenhofer UK et al (2019) In vivo accuracy of tooth surface reconstruction based on CBCT and dental MRI-A clinical pilot study. Clin Oral Implants Res 30:920-927.

The previous study assessed the spatial accuracy of tooth-surface reconstructions based on dental MRI and CBCT data. Altogether, 18 study subjects of the current patient cohort participated in the previous study as well. Imaging of tooth surfaces within MRI data resembles an essential intermediate step in guided dental implant surgery. Tooth surfaces are used for co-registration of spatially highly accurate oral surface data (derived from intraoral scans or digitalized models) and CBCT data. The previous publication introduced a new method to visualize tooth surfaces in dental MRI and compared it's accuracy with the accuracy of CBCT, using a digitalized model as reference. It evaluated, however, not the accuracy and reliability of dental MRI for implant planning.

\section{Methodology \\ - prospective \\ - diagnostic study \\ - performed at one institution}

Open Access This article is licensed under a Creative Commons Attribution 4.0 International License, which permits use, sharing, adaptation, distribution and reproduction in any medium or format, as long as you give appropriate credit to the original author(s) and the source, provide a link to the Creative Commons licence, and indicate if changes were made. The images or other third party material in this article are included in the article's Creative Commons licence, unless indicated otherwise in a credit line to the material. If material is not included in the article's Creative Commons licence and your intended use is not permitted by statutory regulation or exceeds the permitted use, you will need to obtain permission directly from the copyright holder. To view a copy of this licence, visit http://creativecommons.org/licenses/by/4.0/.

\section{References}

1. Branemark PI, Adell R, Breine U, Hansson BO, Lindstrom J, Ohlsson A (1969) Intra-osseous anchorage of dental prostheses. I. Experimental studies. Scand J Plast Reconstr Surg 3:81-100 
2. Buser D, Sennerby L, De Bruyn H (2017) Modern implant dentistry based on osseointegration: 50 years of progress, current trends and open questions. Periodontol 2000 73:7-21

3. Elani HW, Starr JR, Da Silva JD, Gallucci GO (2018) Trends in dental implant use in the U.S., 1999-2016, and projections to 2026. J Dent Res 97:1424-1430

4. D'Haese J, Ackhurst J, Wismeijer D, De Bruyn H, Tahmaseb A (2017) Current state of the art of computer-guided implant surgery. Periodontol 2000 73:121-133

5. Katsoulis J, Pazera P, Mericske-Stern R (2009) Prosthetically driven, computer-guided implant planning for the edentulous maxilla: a model study. Clin Implant Dent Relat Res 11:238-245

6. Tzerbos F, Sykaras N, Tzoras V (2010) Restoration-guided implant rehabilitation of the complex partial edentulism: a clinical report. J Oral Maxillofac Res 1:e8

7. Sanz M, Noguerol B, Sanz-Sanchez I et al (2019) European Association for Osseointegration Delphi study on the trends in implant dentistry in Europe for the year 2030. Clin Oral Implants Res 30:476-486

8. Jacobs R, Salmon B, Codari M, Hassan B, Bornstein MM (2018) Cone beam computed tomography in implant dentistry: recommendations for clinical use. BMC Oral Health 18:88

9. Pauwels R, Zhang G, Theodorakou C et al (2014) Effective radiation dose and eye lens dose in dental cone beam CT: effect of field of view and angle of rotation. Br J Radiol 87:20130654

10. Memon A, Rogers I, Paudyal P, Sundin J (2019) Dental X-rays and the risk of thyroid cancer and meningioma: a systematic review and meta-analysis of current epidemiological evidence. Thyroid 29: $1572-1593$

11. Gray CF, Redpath TW, Smith FW (1998) Low-field magnetic resonance imaging for implant dentistry. Dentomaxillofac Radiol 27: 225-229

12. Hassfeld S, Fiebach J, Widmann S, Heiland S, Muhling J (2001) Magnetic resonance tomography for planning dental implantation. Mund Kiefer Gesichtschir 5:186-192

13. Pompa V, Galasso S, Cassetta M, Pompa G, De Angelis F, Di Carlo S (2010) A comparative study of magnetic resonance (MR) and computed tomography (CT) in the pre-implant evaluation. Ann Stomatol (Roma) 1:33-38

14. Goto TK, Nishida S, Nakamura Y et al (2007) The accuracy of 3dimensional magnetic resonance 3D vibe images of the mandible: an in vitro comparison of magnetic resonance imaging and computed tomography. Oral Surg Oral Med Oral Pathol Oral Radiol Endod 103:550-559

15. Aguiar MF, Marques AP, Carvalho AC, Cavalcanti MG (2008) Accuracy of magnetic resonance imaging compared with computed tomography for implant planning. Clin Oral Implants Res 19:362365

16. Imamura H, Sato H, Matsuura T, Ishikawa M, Zeze R (2004) A comparative study of computed tomography and magnetic resonance imaging for the detection of mandibular canals and crosssectional areas in diagnosis prior to dental implant treatment. Clin Implant Dent Relat Res 6:75-81
17. Flugge T, Ludwig U, Hovener JB, Kohal R, Wismeijer D, Nelson K (2020) Virtual implant planning and fully guided implant surgery using magnetic resonance imaging-proof of principle. Clin Oral Implants Res. https://doi.org/10.1111/clr.13592

18. Cohen JF, Korevaar DA, Altman DG et al (2016) STARD 2015 guidelines for reporting diagnostic accuracy studies: explanation and elaboration. BMJ Open 6:e012799. https://doi.org/10.1136/ bmjopen-2016-012799

19. Hilgenfeld T, Juerchott A, Deisenhofer UK et al (2019) In vivo accuracy of tooth surface reconstruction based on CBCT and dental MRI-a clinical pilot study. Clin Oral Implants Res 30:920-927

20. Buser D, Martin W, Belser UC (2004) Optimizing esthetics for implant restorations in the anterior maxilla: anatomic and surgical considerations. Int J Oral Maxillofac Implants 19(Suppl):43-61

21. Gallucci GO, Hamilton A, Zhou W, Buser D, Chen S (2018) Implant placement and loading protocols in partially edentulous patients: a systematic review. Clin Oral Implants Res 29(Suppl 16):106-134

22. Li G, Nittka M, Paul D, Lauer L (2011) MSVAT-SPACE for fast metal implants imaging. Proc ISMRM 19:3171

23. Hilgenfeld T, Prager M, Heil A et al (2017) PETRA, MSVATSPACE and SEMAC sequences for metal artefact reduction in dental MR imaging. Eur Radiol 27:5104-5112

24. Landis J, Koch G (1977) An application of hierarchical kappa-type statistics in the assessment of majority agreement among multiple observers. Biometrics 33:363-374

25. Prager M, Heiland S, Gareis D, Hilgenfeld T, Bendszus M, Gaudino C (2015) Dental MRI using a dedicated RF-coil at 3 Tesla. J Craniomaxillofac Surg 43:2175-2182

26. Sedlacik J, Kutzner D, Khokale A et al (2016) Optimized $14+1$ receive coil array and position system for $3 \mathrm{D}$ high-resolution MRI of dental and maxillomandibular structures. Dentomaxillofac Radiol 45:20150177

27. Huber FA, Schumann P, von Spiczak J et al (2020) Medicationrelated osteonecrosis of the jaw-comparison of bone imaging using ultrashort Echo-time magnetic resonance imaging and cone-beam computed tomography. Invest Radiol 55:160-167

28. Behneke A, Burwinkel M, Behneke N (2012) Factors influencing transfer accuracy of cone beam CT-derived template-based implant placement. Clin Oral Implants Res 23:416-423

29. Ersoy AE, Turkyilmaz I, Ozan O, McGlumphy EA (2008) Reliability of implant placement with stereolithographic surgical guides generated from computed tomography: clinical data from 94 implants. J Periodontol 79:1339-1345

30. Kuhl S, Payer M, Zitzmann NU, Lambrecht JT, Filippi A (2015) Technical accuracy of printed surgical templates for guided implant surgery with the coDiagnostiX software. Clin Implant Dent Relat Res 17(Suppl 1):e177-e182

Publisher's note Springer Nature remains neutral with regard to jurisdictional claims in published maps and institutional affiliations. 